zur Diskussion zu stellen. Systematische begriffliche Klärungen, z.B. zum Begriff des "virtuellen Geldes", werden nur sporadisch versucht, geben dem Band jedenfalls keinen einheitlichen Rahmen. Für eine grundlegende Einführung in das Thema ist er deshalb nicht geeignet. Wer aber Argumente sucht in der Diskussion über die Chancen und Risiken der neuen elektronischen Zahlungssysteme in einem breiteren gesellschaftlichen Kontext, sollte nicht zuletzt diesen Band zu Rate ziehen.

》

\section{Interaktionsraum Internet ... The Final Report}

\section{Rezension des Schlußberichts des Projekts "Interaktionsraum Internet: Netzkultur und Netzwerkorganisation in offenen Datennet- zen" von Knud Böhle, ITAS}

Wer nicht weiß was ein Lurker, ein Sysop, ein Luser, ein DAU oder ein Spammer ist, wird nebenbei auch dies in dem bemerkenswerten, knapp 140 Seiten starken Schlußbericht des Projekts "Interaktionsraum Internet. Netzkultur und Netzwerkorganisation in offenen Datennetzen" erfahren können. Bereits 1994 hatte sich die Projektgruppe "Kulturraum Internet" unter Beteiligung der TU Berlin in der Abteilung "Organisation und Technikgenese" des Wissenschaftszentrums Berlin gebildet, deren Projektvorhaben in den Jahren 1996 - 1998 von der Volkswagen-Stiftung gefördert wurde (ein erster Bericht zu diesem Projekt ist erschienen in den TA-Datenbank-Nachrichten Nr.1, April 1997, S. 15 ff.).

Die Projektgruppe wählte einen ethnographischen Ansatz, um die Netzwelt "von innen heraus" zu beschreiben (S. 7). Die Methode der Wahl war die teilnehmende Beobachtung, die auch in Computernetzen möglich ist und dort in neuartigen Formen praktiziert werden kann. Für die empirische Erforschung dieses Kulturraums ist allerdings "eine netztechnische Ausstattung und Praxis" (S. 8) Voraussetzung - konkret z.B. der Betrieb eines Servers im Internet oder die Etablierung eines E-Mail-Diskussionsforums. Dadurch werden Beschreibungen ermöglicht, die durch ihren Stil, der Raum für viele Originalzitate läßt, athmosphärisch dicht sind, ohne das Forschungsobjekt bloßzustellen. Da die Autorinnen die Ergebnisse ihrer Erkundungen zudem im Lichte theoretischer Debatten (Technikgenese, Medientheorie, Governance) reflektieren, gelingt ihnen auch der Schritt von der Ethnographie zur politischen Soziologie.

Der Bericht versammelt drei sich ergänzende Fallstudien. In der ersten Fallstudie wird die Entwicklung des Betriebssystems Unix, das einige Zeit praktisch konkurrenzlos auf Internet-Servern zum Einsatz kam, und die umgebende "Unixkultur" untersucht, die die Prinzipien der späteren Internetkultur mitgeprägt hat: wesentlich die kooperative Softwareentwicklung, die Maxime einer offenen Netzarchitektur, das Ideal freien Datenflusses und freier Software. Ebenfalls noch im Rahmen dieser Fallstudie wird untersucht, wie dieses Ethos seinen Niederschlag in der "Netiquette", den Verhaltens- und Kommunikationsregeln im Internet, gefunden hat. Wie in den weiteren Fallstudien auch, geht es nicht darum, verloren gehende paradiesische Zustände zu beschwören, sondern vielmehr darum, Statik und Dynamik der Internetkultur unter dem Eindruck des ungebremsten Wachstums des Netzes und des Ansturms kommerzieller Interessen zu begreifen.

In der zweiten Fallstudie werden die technischen Standards bzw. deren Weiterentwicklung - einschlägiges Thema der GovernanceForschung, hier des Internet - zum Gegenstand gemacht mit der Besonderheit, daß die Forschungsstrategie "bottom up" verfährt. Die Diskussionen um die Reform des grundlegenden Internetprotokolls (IP) und die Herausbildung von entsprechenden Standardisierungsvorschlägen selbst werden verfolgt. Dabei steht die IETF, die Internet Engineering Task Force, das wohl immer noch wichtigste Normungsgremium des Internet, im Mittelpunkt. Die Kultur dieser Ingenieure, die weniger an grauen Sandalen und weißen Socken als am obligatorischen T-Shirt zu erkennen sind, ist durch ein Selbstverständnis ausgezeichnet, das einer der Protagonisten einmal auf folgende Formel brachte: "We reject presidents, kings and voting, we believe in rough consensus and running code" (S. 53). Diese Kultur ist ebenfalls mit dem Wachstum des Internet und der zu- 
nehmenden Bedeutung mächtiger Firmen konfrontiert. Wichtig an dieser Fallstudie ist auch, daß sie nicht nur aufzeigen kann, wie schwer es beim jetzigen Stand der Netzentwicklung geworden ist, Normen noch allgemein verbindlich durchzusetzen, sondern auch, wie stark mit der technischen Normung bereits implizit eine keineswegs interessenneutrale "Raumordnungspolitik" einhergeht.

Was man sich in den beiden angesprochenen Fallstudien noch genauer ausgeleuchtet gewünscht hätte, ist gewissermaßen die Bedingung der Möglichkeit der "Unix- bzw. IETFKultur" und deren Selbstverständnis als quasi "freischwebend". Sowohl über die materielle Absicherung, berufliche Verankerung und möglicherweise auch Abhängigkeit der Ingenieure als auch über die Unternehmensstrukturen, die ihren Mitarbeitern den nötigen Spielraum ermöglichen, hätte man gerne mehr erfahren.

Der dritte Teil untersucht das inzwischen zum "Massenmedium" expandierte Usenet, auf dem im Sommer 1998 täglich 200.000 Artikel (bei über 70.000 Gruppen) verbreitet wurden (S. 84). Weil am Usenet Kommunikationskultur, Medienentwicklung und Governance in einem interessierten, wurden speziell die Gruppen im Usenet, die das Medium selbst und seine Entwicklungsprobleme zum Inhalt haben, beforscht. Interessant ist, um hier nur einen Punkt herauszustellen, die These, mit der diese Fallstudie abschließt: Wird allgemeinhin angenommen, daß ein Medium hoch entwickelt ist, wenn sich die Kommunikationstechnik nicht mehr bemerkbar macht oder nicht mehr eigens wahrgenommen wird, könnte das Usenet für einen neuen Typus "telematischer Interaktivität" (S. 112) stehen, für den das Rauschen des Mediums konstitutiv wäre und der den Nutzern folglich dauerhaft abverlangte, "sich im kommunikativen Handeln der Funktionsweise und Materialität des Mediums selbst" zuzuwenden.

Nach den drei Fallstudien suchen die Autorinnen nach gemeinsamen Befunden. Die folgende Passage ist ein Ausschnitt (S. 113f) daraus, der wichtige Ergebnisse festhält und noch einmal deutlich macht, daß es den Autorinnen nicht um Nostalgie, sondern um die Bestimmung der heutigen Lage zu tun ist, um die "Konsequenzen, die die offene Organisation des Internet für seinen Fortbestand hat:
Skalierungsprobleme, Regulierungsversagen und Bedeutungsverlust einstmals unangefochtener Verhaltensregeln ließen das exponentielle Wachstum des Netzes bald als Vertreibung aus dem Paradies, bald als unmittelbar bevorstehenden Kollaps erscheinen. Zumindest als Echo findet sich die Tendenz zur Krisen- und Katastrophenrhetorik auch in unseren Berichten über das Internet wieder. Allerdings sind die so häufig prophezeiten Zusammenbrüche weder auf der Ebene der Übertragungstechnik, noch im Bereich der Netiquette oder im Usenet eingetreten. Das Internet erweist sich vielmehr als bemerkenswert robust im Umgang mit dem Ansturm auf seine Ressourcen. Wohl erfordert die Transformation vom Wissenschaftstempel zum Massenmedium ihren Preis: Der Zerfall des Usenet in einzelne Verwaltungsbezirke, die sich durch unterschiedliche Kontroll- bzw. Ordnungsformen auszeichnen, ist ein Beispiel dafür. Wachstum und Dezentralisierung des Kommunikationsdienstes lassen netzweit einheitliche Regularien nicht mehr zu. Ein weiteres Beispiel stellt die zunehmende Tendenz zur Deaktivierung einzelner Unixfunktionen wie "finger" oder "remote login" dar. Der ungehinderte Zugriff auf Netzressourcen, wie er für das Wissenschaftsnetz kennzeichnend war, fällt einer schärferen Differenzierung zwischen privaten und öffentlichen Räumen im Netz zum Opfer: Parzellierungsprozesse im offenen Datennetz. Zugangssperren in Form von Firewalls sollen Sites vor Mißbrauch schützen, unterhöhlen aber zugleich die Tradition des Teilens und kollektiven Austauschs. Auch die Neuordnung der Besitzverhältnisse im Adreßraum ist Bestandteil der Transformationsprozesse im Internet. Die Verwaltung einstmals öffentlicher Güter wie der Netzadressen geht über in die Hände der Internet Service Provider - eine Kraft in der Regulierung des Internet, die auf den Ausbau des Leitungsnetzes wie auch die Etablierung neuer Dienste und Übertragungstechniken wachsenden Einfluß gewinnt. Der übertragungstechnisch verteilten Handlungsmacht im Netz steht ein Konzentrationsprozeß auf Seiten der Internetanbieter gegenüber. ...".

Die Transformation des Internet stellt sich jedoch in keinem der Untersuchungsfelder als reine Verlustbilanz dar. Den Untergangsgesängen, die angesichts der Entwicklung des Internet hier und da angestimmt werden, läßt sich entgegenhalten, daß sich die Kommunikationsmöglichkeiten im Netz eher erweitern als verengen. Dies betrifft nicht nur die Zahl der Menschen, Organisationen oder Datenbanken, die über das Internet $\mathrm{zu}$ erreichen sind, sondern auch die Dienste, vermittels derer kommuniziert werden kann. 
Die Studie regt an, weiter darüber nachzudenken, gerade angesichts der Kommerzialisierung des Internet, der zunehmenden Bedeutung des elektronischen Geschäftsverkehrs, des wachsenden Einflusses von Medien- und anderen Konzernen, ob die als ziemlich motivierend, produktiv und leistungsfähig erfahrene Internetkultur bald überholt sein wird, oder ob jemand etwas für ihre Erhaltung tun kann (wer aber, wenn nicht sie selbst). Schutzzonen im Internet wird es kaum geben. Koexistenz und Mischformen von "grassroot"-Impulsen und kommerziellen Interessen sind nicht unwahrscheinlich, denkt man z.B. an Dienstleister im Internet wie amzon.com oder travelocity, die kommerzielle Angebote und privates Engagement zusammenbringen, Softwarefirmen, die Teile ihrer Internetsoftware verschenken oder zur Weiterentwicklung freigeben etc.

Bleibt nachzutragen, daß der Bericht von einer CD-ROM begleitet wird, die den Abschlußbericht in elektronischer Form als Hypertext zugänglich macht - bereichert um nicht weniger als 50 Publikationen und Papers der Projektgruppe, sowie das vollständige Archiv der projektbegleitend betriebenen Mailingliste "Netzforum", die eine Zeit lang ein wichtiges Kommunikationsforum für Internetangelegenheiten in Deutschland war. Dazu kommen einiges multimediale Material und zahlreiche Links auf andere Projekte, so daß auch Einblicke in die Projektgeschichte und die Vertiefung bestimmter Argumente möglich werden. Dasselbe Angebot wird auch im WWW gemacht (http://duplox.wz-berlin.de). Die Abbildung zeigt die Übersichtsseite des elektronischen Angebots.

\section{Bibliographische Angaben}

Sabine Helmers, Ute Hoffmann und Jeanette Hofmann: Internet... the Final Frontier: eine Ethnographie. Schlußbericht des Projekts "Interaktionsraum Internet: Netzkultur und Netzwerkorganisation in offenen Datennetzen". Wissenschaftszentrum Berlin: Berlin 1998 (WZB Discussion Paper FS II 98-112)

Der Bericht kann schriftlich beim Presse- und Informationsreferat des WZB, Reichpietschufer 50, 10785 Berlin bestellt werden. Ein ausgefüllter Adreßaufkleber sowie eine Briefmarke im Wert von DM 1,00 je angefordertem Bericht sind beizulegen. Bei Bestellungen aus dem Ausland ist ein "Coupon Résponse International" mitzuschicken.

\section{PRONEKTGRUPPE KULIURRAUM INTERNET}

im Wissenschaftszentrum Berlin für Sozialforschung

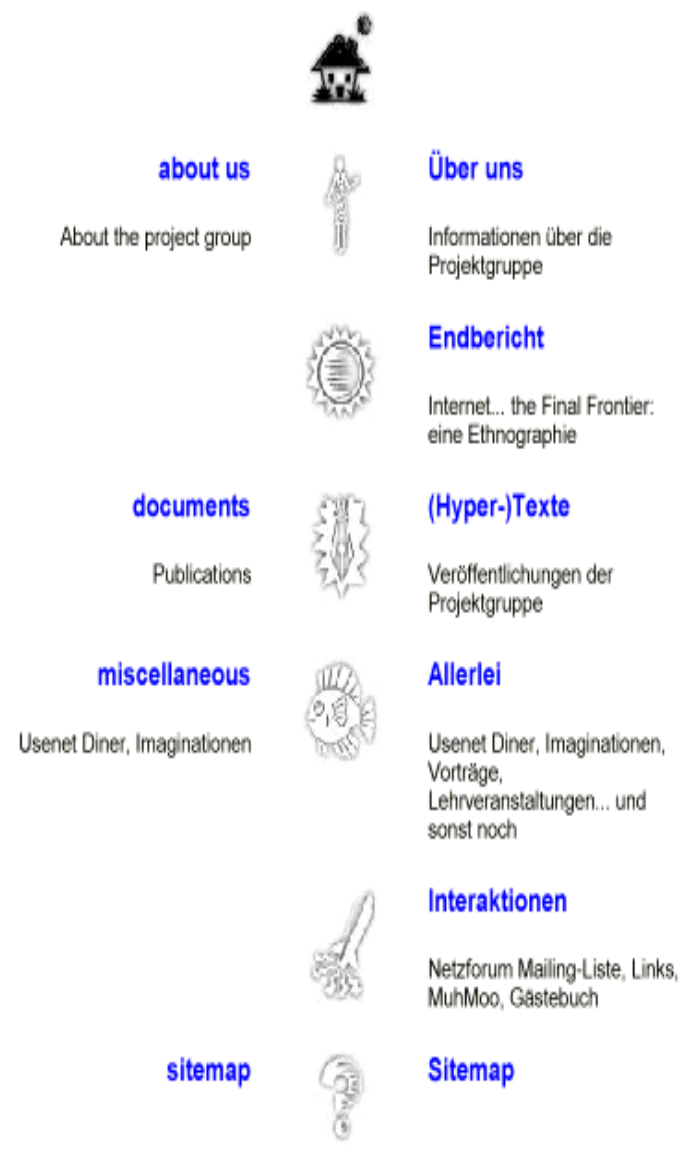

Copynght 1994-1998 Projektoguppe "Kuturraum Internet", clo Wiscenschathzzentrum Berin fôr Sozialtorschung (WZB) Rechpietschufer 50, 10785 Berin. Telefon: (030) 25491 - 207; Fax: (030) 25491 - 209

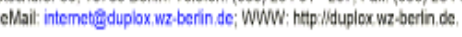

